\title{
Journal of Environmental Engineering and Science: Referees 2019
}

The following is a list of referees who have reviewed papers for Journal of Environmental Engineering and Science between 1 December 2018 and 30 November 2019. The Institution of Civil Engineers (ICE) is very grateful for their assistance.

We are continually looking for suitable reviewers for papers submitted to Journal of Environmental Engineering and Science. Papers published in the ICE journals must be submitted to at least two independent referees to judge accuracy, style, impact, importance and interest.

If you are interested in reviewing articles on any topic related to infrastructure asset management, please submit your name, qualifications or CV, and areas

Bassim Abbassi

Mushtaque Ahmed

Sirajuddin Ahmed

Mostafa Ali

James Alleman

Abdalrahman Alsulaili

Ata Amini

Wajahat Annayat

Olga Anne

Sayali Apte

Hazi Azamathulla

Mahad Baawain

Ravichandar Babarao

Codruta Badaluta

Ashish Borgaonkar

Gerd Bruss

Mengqiu Cao

Daniel Cassidy
May Chan

Theo Chineke

Lansana Coulibaly

Bipro Dhar

Di Di Zhang

Yan-Jun Du

Seda Durukan

Naser Ebadati

Marwa Eltayeb

David Farrusseng

Kazi Fattah

Alessandra Lee Firmo

Qing-Long Fu

Fawzi Gharagheer

Rajesh Goyal

Yelda Hangun-Balkir

Mehdi Hassanshahian

Hiroshan Hettiarachchi

Janitha Hewa Batagoda of expertise. We are in need of individuals who will agree to review papers in a timely fashion (within 3 to 4 weeks of receipt) and provide confidential feedback to the Editorial Advisory Panel concerning the quality of the paper and any suggested revisions that would be appropriate.

If you are such a person, please contact Ben Ramster (tel.: +44 (0)207 665 2242; e-mail: ben.ramster@icepublishing.com) for more information on the referee process.
Shaini Hewage

Harada Hiroyuki

Vlassios Hrissanthou

Veeriah Jegatheesan

Santosh Kadapure

Anand Karre

Mohammed Isah Kimpa

Denise-Penelope Kontoni

Keerthivasan Krishnamoorthy

Alban Kuriqi

Nirban Laskar

Yaal Lester

Michael Lucasml

Ravichandran M.

Sandip Mali

Hanapi Mat

Yasuhiro Mitani

Jean Aimé Mono

N. M. Mubarak
Soumyadeep Mukhopadhyay Emily O'Donnell

Rajan Ray

S. N. Reddy

Balasubramani S. P.

Takashi Sakamaki

Khamis Sayl

Ana Segura Carnicero

Xiaonan Shi

Aline Auxiliadora Tireli

Joseph Tripura

Zahoor Ullah

Yi Wang

Priscilla Williams

Zaher Yaseen

Pengwei Zhang 